Original Research

\title{
How to Prioritize Voluntary Dietary Modification
}

\section{Gidon Eshel *}

Bard College, Annandale-on-Hudson, New York 12504-5000, U.S.A.; E-Mail: geshel@gmail.com.

* Correspondence: Gidon Eshel; E-Mail: geshel@gmail.com.

Academic Editor: Zed Rengel

Special Issue: Climate Change and Land

Adv Environ Eng Res

2020, volume 1 , issue 4

doi:10.21926/aeer.2004005
Received: August 03, 2020

Accepted: December 15, 2020

Published: December 25, 2020

\begin{abstract}
Environmentally motivated voluntary diet modifications are the focus of much research and public discourse. Yet the nuanced multi-faceted, multi-dimensional nature of agricultureearth interactions, and limited public environmental knowledge likely combine to undermine the efficacy of environmentally motivated dietary shifts, squandering limited good will. To counter this, here I devise two related indices for crudely estimating importance of various environmental impacts of alternative dietary choices. Based on the devised indices (whichcoarse, simple and inexhaustive-are best viewed as preliminary assessment) I find that in the U.S., soil conservation and water use in the arid west may well dominate the environmental impacts of food, easily eclipsing greenhouse gas emissions, and that of a limited considered subset of possible dietary shifts, replacing beef with tofu, some legumes, and some cereals are the most beneficial modifications.
\end{abstract}

\section{Keywords}

Resource use; environmental optimization; dietary shifts 


\section{Introduction}

While appreciation of the vast scope of some of our environmental challenges is wide, governmental action is mostly limited, sporadic, and timid, falling well short of our needs. Many individuals thus seek to make some voluntary behavioral changes that they perceive as environmentally important.

This is clearly an opportunity; absent concerted governmental efforts on scales consistent with the magnitude of the task, the collective impact of numerous individuals making small changes that yield modest improvements is attractive. It is also a challenge, stemming from the uneven distribution of environmental knowledge; environmentally untrained persons are not likely to choose the optimal voluntary dietary changes.

While prevalent throughout human environmental agency, environmental suboptimality in dietary choices is all the likelier because farming is foreign to most consumers, whose ideas about modern food production are often vague and unrealistic. This leads to misplaced efforts that may yield minor improvements but overlook more effective interventions. Striving to overcome this, here I devise two related simple indices for estimating the importance of environmentally motivated considered dietary interventions. Because they are simple and designed to offer easy implementation, the indices and the results below are best viewed as preliminarily suggestive rather than definitive. In addition, broader societal objectives may well call for usurping conclusion drawn from these indices. For example, in the U.S., the former Soviet Union, or Israel, to name but a few obvious examples, agricultural settlements have been widely used as means to such broader societal objectives as population dispersion and spatial distribution or affirming political territorial stakes, completely disregarding often devastating environmental consequences. This paper does not address such non-environmental goals.

\section{Methods}

For environmental burden $k$ (which can be, e.g., water use, or greenhouse gas emissions), the non-negative, real importance index is

$$
I_{k} \sim \sigma_{k} \rho_{k}
$$

in which $I$ is the estimated dimensionless savings of environmental resource $k$ various alternatives can deliver. The relational symbol $(\sim)$ highlights that $I$ is a rough estimate, conceptually akin to formal scaling of governing equations [1], and $\sigma_{k}$ is the share of agriculture in the total national budget of environmental burden $k$ (e.g., because-as the Supplementary Information (hereafter SI) shows - food production accounts for $10-15 \%$ of total national greenhouse gas emissions, $\sigma_{\mathrm{GHG}} \approx$ 0.12). Finally, $\rho$ is a representative quantitative estimate of the full range of resource uses by realistically interchangeable alternative. In practice, we calculate this range using $\rho_{k}=b_{i k} / b_{j k}$, where $b_{i k}$ is the $k$ th burden (e.g., reactive nitrogen, water, or cropland use) exerted by alternative $i$, with $i$ and $j$ chosen so as to span the full range of resource use by two specific realistically interchangeable alternatives. For example, if emissions of various interchangeable alternative food items span 3 to $6 \mathrm{~kg} \mathrm{CO}_{2 \text { eq }} \mathrm{g}^{-1}$, then $\rho_{\mathrm{GHG}}=2$.

The rationale behind $I$ is as follows. To significantly reduce the dietary environmental burdens of a person considering a given environmentally motivated dietary shift, the considered shift must 
simultaneously satisfy two conditions. First, corresponding to $\sigma$, agriculture or diet must account for a sizable portion of the current total national burden. For example, because agriculture uses $\lesssim 1 \%$ of total U.S. energy [2] (i.e., $\sigma_{\text {energy }} \approx 0.01$ ), even if-implausibly-all Americans switched to a zero energy diet, we would still require $99 \%$ of the energy we currently use. Low energy agricultural alternatives can thus reduce our current total energy by at most 1\%, which most impartial observers would likely deem minute and inconsequential. This indicates the fruitlessness of this general direction, which stands to reduce overall energy use only trivially even with draconian, manifestly unrealistic dietary shifts. More generally, a demanding behavioral modification for the sake of environmental betterment requires considerable commitment, and if the potential benefits are small, one may wish to devote their efforts to other, more efficacious changes.

Second, corresponding to $\rho$, use of the examined resource per unit product must vary widely. A glaring example would be replacing beef with some cereal or pulse alternatives, which can reduce land use per g protein about 100-fold [3], i.e., $\rho_{\text {land }}=100$. With such broad ranges, viable alternatives to today's state (the agricultural practices or food items the alternatives replace) can use far less or far more of the examined resource, permitting significant savings. Because both conditions are necessary for large expected impacts, $I$ multiplicatively combines them so that when only one holds (e.g., when $\sigma=1$ and $\rho=0$ ), I vanishes, as $\rho=0$ dictates.

The index $I$ also forms the basis for another dimensionless index, measuring the environmental desirability of the $i \rightarrow j$ replacement. This index [4] is given by

$$
\delta_{i \rightarrow j}=\sum_{k=1}^{K}\left[I_{k} \frac{b_{i k}-b_{j k}}{b_{\max , k}-b_{\min , k}}\right]
$$

where $k \in[1, K]$ is the environmental burden index, $b_{i k}$ is the use of burden $k$ by option or alternative $i$, and the burden-specific extrema in the denominator are calculated over all food items available. The larger the normalized burden savings (the quotient), and the larger the importance index $I$ of the burden, the larger the contribution of burden $k$ to the sum, and the more desirable the $i \rightarrow j$ replacement becomes. Note that as formulated, $\delta_{i \rightarrow j}<0$ is possible, when the replacements systematically require more resources than the item they replace, indicating undesirability. We discuss the environmental desirability index, and its rationale and applications, below.

\section{Results}

Figure 1 presents a few derived $I$ values. Note that evaluating the ranges $\rho$ needed for quantifying various $I$ values may involve quite physically distinct processes. For example, while the metrics shown by the four right bars in Figure 1 reflect ranges $\rho$ derived from the differences between individual food items that can reasonably replace one another, the leftmost metric$I_{\text {soil }}$-is derived by contrasting conventional and soil-sparing agricultural practices (see the SI). This mixed methodology addresses the wide range of natural geophysical processes agriculture perturbs, which is a key challenge in agricultural and diet optimizations [4,5]. 


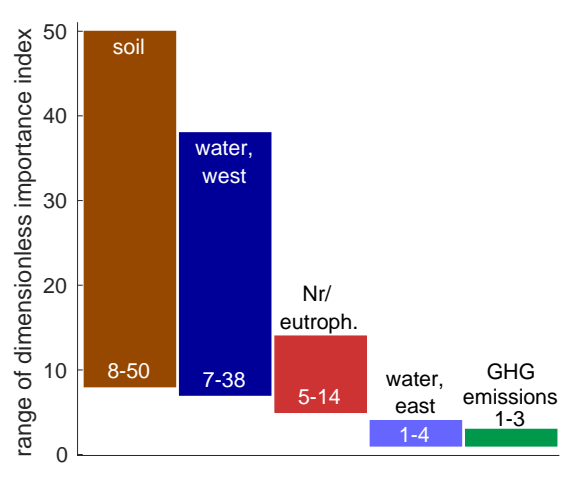

Figure 1 Importance index I for five environmental burdens (with ranges derived in the SI). Soil estimates contrast conventional and soil-sparing alternative practices, while other estimates reflect ranges various food items span. All are actionable in most supermarkets. For water, we distinguish the arid west, from which much of the U.S. produce originates, from the rainy portion of the U.S. (east of $\approx 95^{\circ} \mathrm{W}$ ).

Figure 1 suggests that water is of minimal concern [6] when used in the eastern half of the U.S. (light blue bar), but a major one when used in the arid west [7] (dark blue bar), most importantly the Central Valley of California [8]. (A complementary calculation, addressing groundwater withdrawal, would further accentuate [9] this east-west lop-sidedness [10].) This meridional dichotomy is intuitive, because it is the agricultural manifestation of the widely appreciated eastwest hydrometeorological boundary [6] longitude $100^{\circ} \mathrm{W}$ delineates in the contiguous U.S. It is also critically important for dietary decisions, because water is far scarcer west of $100^{\circ} \mathrm{W}$, especially in the Central Valley of California [11], and because most fresh produce consumed in the U.S. originates in this valley. This highlights the utility of $I$ as a tool for dietary-environmental decision making.

Likely most surprising for many would be the small $I_{\text {GHG. }}$. This reflects the small portion of total U.S. emissions for which agriculture accounts. To be clear, this result by no means justifies disregarding greenhouse gas considerations in dietary choices. Because no single solution is likely to solve anthropogenic climate change, our defining environmental challenge, it will require numerous small improvements instead [12]. Consequently, a sector responsible for $10-15 \%$ of emissions cannot be ignored, especially given the large improvement potential due to the wide emission range $\rho_{\mathrm{GH}}$ various nutritionally interchangeable food items span [3, 13-15].

Probably less surprising is the considerable potential for agricultural eutrophication reduction (red bar). With agriculture dominating national nutrient portfolios, and with considerable range various nutritionally interchangeable food items span, this large potential is intuitive.

Most remarkable is the towering soil sparing potential of dietary choices (brown bar). It reflects the alignment of agricultural dominance over the total national soil loss with conventional agriculture losses being 5-50 times faster than under conservation agriculture. Figure 1 suggests that over long timescales, soil conservation may well be the most existential dimension of environmentally mindful agriculture.

Next, we turn to the environmental desirability index $\delta_{i \rightarrow j}$ (Eq. 2). This index cogently combines and unifies various environmental burden differences a specific considered dietary replacement is expected to yield. The challenge arises because in general environmental burdens are measured in distinct units, and are governed by different geophysical processes (often characterized by distinct 
timescales), complicating their comparison and rendering it at least partly subjective. The comparison becomes all the more imperative and challenging when various burdens of a given considered dietary shift mutually contradict. For example, consider a protein conserving replacement of almonds with oats. Per g protein, oats are about tenfold more greenhouse intensive $[3,16]-24$ vs. $2.5 \mathrm{~g} \mathrm{CO}_{2 \text { eq }}$-but require only $5 \mathrm{~L}$ of water (g protein) $)^{-1}$ to almonds' 26. Critically, because most U.S. almonds originate in the Central Valley while oats mostly in the northern Midwest and Plains, almonds use scarcer water. Emission and water use minimization thus conflict in this case, concealing the optimal path.

To overcome this apparent stalemate, Figure 2 shows dimensional individual environmental consequences of replacing each of the animal items with protein conserving masses of each of the considered plant alternatives (a-c), and the corresponding desirability $\delta_{i \rightarrow j}$ for $i=[1,5]$ livestock categories and $j=[1,16]$ relatively protein rich plant items $(\mathrm{d})$. These results only consider eutrophication, greenhouse gas emissions and water use, and future observation may well further numerically refine them. Yet they demonstrate the power of $I$ and $\delta$ in guiding decisions, highlighting the importance of replacing beef. Even when grown in the arid west, most plant alternatives to beef still outperform the beef they replace. Conversely, benefits of replacing other livestock with plant alternatives depend sensitively on the details.
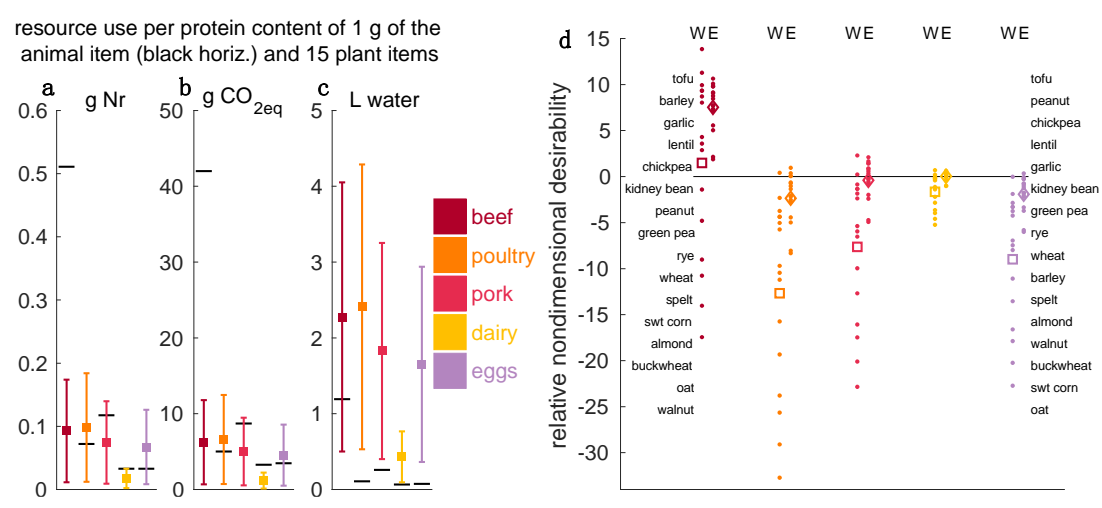

Figure 2 resource use per unit protein by the livestock categories (horizontal black lines) and mean \pm 1 standard deviation (solid squares and whiskers respectively) of 16 relatively high protein plant items. To be clear, the squares and whiskers in a-c reflect only dimensional resource use by plants, but are colored by the animal item they replace while conserving protein. Panel $d$ shows the corresponding desirability indices, presenting the individual desirability indices (dots) and their respective means (open symbols) for replacing each of the livestock categories with plant alternatives. The items in order of desirability (led by tofu, barley, and protein-rich legumes) are given on the left and right for the western and eastern U.S. (distinguished by " $W$ " and " $E$ " and by open square and diamond markers). In $d$, plant environmental costs are inflated by $30 \%$ relative to the original data $[14,15]$, to reflect soil sparing alternative production. That is, if in our original resource use data a ton of a given plant item requires 1 ha-yr of cropland, here we replace this value with 1.3 ha-yr, to allow for the distinct possibility that the soil sparing alternative practice suppresses yields by $30 \%$. This inflation of the resource use by plants renders the resource savings estimates of the Figure a conservative lower bound on what can be saved by the animal-to-plant item replacements considered. 


\section{Discussion and Conclusions}

The importance index I combines agriculture's dominance over the total national use of a given environmental resource with a realistic range of such uses spanned by alternative choices. The index uses this combination to quantify the importance of diet-related individual environmental burdens for motivating voluntary individual dietary shifts or alternative agricultural policies. In doing so, $I$ also facilitates relative ranking of entertained dietary or policy changes, and identifies those that stand to deliver the most environmental returns. While similar methodology may be relevant to other factors impacted by diet (notably qualitative social considerations), or to other (diet-unrelated) realms, such generalizations are not straightforward, must each be carefully individually considered, and are thus not covered here.

We turn to the desirability index $\delta$ next. By appeal to Eq. 2, clearly only replacements associated with $\delta_{i \rightarrow j}>0$ are objectively desirable; $\delta_{i \rightarrow j}<0$, which arises when the replacements systematically require more resource than the item they replace, indicates increased combined normalized weighted resource use. Figure $2 \mathrm{~d}$ may thus appear perplexing, as it shows that only replacing beef, and replacing pork with plant alternatives grown in the eastern U.S., are decidedly mostly desirable. Do these results call into question the widely held view that shunning animalbased food items is an environmental necessity [17]?

For beef, the answer is an emphatic no; replacing beef with plant items is almost universally environmentally beneficial, often dramatically so. As for other animal items, the strict assumptions I make here are key. Figure 2 does show that, assuming 30\% lower plant item yields due to deployment of soil sparing practices and requiring exact protein mass conservation, plant replacements are not always environmentally beneficial. Yet even with those stringent requirements, replacing poultry, to choose an item particularly ubiquitous in the U.S. diet, with tofu, peanuts, chickpeas and lentils all still reduce combined normalized weighted resource use, conformal with the prevailing view. More generally, if replacement plant items are grown in the eastern U.S., 16, 4, 8, 11 and 1 plant replacements (out of the 16 considered) offer overall environmental benefits when replacing beef, poultry, pork, dairy and eggs respectively while conserving protein. On the other hand, because all plant items require more water per g protein than poultry, pork, dairy and eggs, and some require even more than beef (see Figure $2 \mathrm{c}$ ), and because the importance index $I$ for water in the west is 2-12 times larger than the other three $I$ values, western water needs completely dominate the sum (Eq. 2).

These findings illustrate the combined power of $I$ and $\delta$ for guiding putative policy options considered. They highlight, e.g., the unsustainability of the U.S. near exclusive reliance on the Central Valley for fresh produce. They also reiterate and reemphasize the unsustainability of beef, and augment the nutritional desirability of legumes as meat alternatives [18] with a complementary environmental desirability. Some may also well take the low rankings and $\delta \ll 0$ values of such nuts as almonds or walnuts (Figure 2) as indicating that California's virtual monopoly over producing these nuts - which can also be grown [19] elsewhere in the U.S. (e.g., the southeast) where water scarcity is minimal-serves us poorly. Finally, the analysis also reveals that despite the easily justifiable dominance of climate change over the environment-diet discourse, it is in fact soil erosion and water use in arid or semi-arid locales (notably the Central Valley, the origin of most U.S. nuts and fresh produce) that should dominate our food-related environmental conversation. 


\section{Author Contributions}

Gidon Eshel did all the research work of this study.

\section{Competing Interests}

The author has declared that no competing interests exist.

\section{References}

1. Pedlosky J. Geophysical fluid dynamics. 1st ed. Berlin: Springer-Verlag; 1979.

2. U.S. jet fuel consumption during the week of Thanksgiving was about half of last year's [Internet]. Washington: Energy Information Administration; 2020. Available from: https://www.eia.gov/todayinenergy/detail.php?id=46316.

3. Eshel G, Shepon A, Makov T, Milo R. Land, irrigation water, greenhouse gas and reactive nitrogen burdens of meat, eggs, and dairy production in the United States. Proc Natl Acad Sci U S A. 2014; 111: 11996-12001.

4. Eshel G. A geophysical foundation for alternative farm policy. Environ Sci Technol. 2010; 44: 3651-3655.

5. Eshel G, Martin PA. Geophysics and nutritional science: Toward a novel, unified paradigm. Am J Clin Nutr. 2009; 89: 1710S-1716S.

6. Seager R, Lis N, Feldman J, Ting M, Williams AP, Nakamura J, et al. Whither the 100th Meridian? The once and future physical and human geography of America's arid-humid divide. Part I: The story so far. Earth Interact. 2018; 22: 1-22.

7. Cook BI, Ault TR, Smerdon JE. Unprecedented 21st century drought risk in the American Southwest and Central Plains. Sci Adv. 2015; 1: e1400082.

8. Pauloo RA, Escriva-Bou A, Dahlke H, Fencl A, Guillon H, Fogg GE. Domestic well vulnerability to drought duration and unsustainable groundwater management in California's Central Valley. Environ Res Lett. 2020; 15: 044010.

9. Ojha C, Shirzaei M, Werth S, Argus DF, Farr TG. Sustained groundwater loss in California's Central Valley exacerbated by intense drought periods. Water Resour Res. 2018; 54: 44494460.

10. Perrone D, Rohde MM. Benefits and economic costs of managed aquifer recharge in California. San Francisco Estuary Watershed Sci. 2016; 14.

11. Alam S, Gebremichael M, Li R, Dozier J, Lettenmaier DP. Can managed aquifer recharge mitigate the groundwater overdraft in California's Central Valley? Water Resour Res. 2020; 56: e2020WR027244.

12. Creutzig F, Fernandez B, Haberl H, Khosla R, Mulugetta $Y$, Seto KC. Beyond technology: Demand-side solutions for climate change mitigation. Annu Rev Environ Resour. 2016; 41: 173-198.

13. Shepon A, Eshel G, Noor E, Milo R. Energy and protein feed-to-food conversion efficiencies in the US and potential food security gains from dietary changes. Environ Res Lett. 2016; 11: 105002.

14. Eshel G, Stainier P, Shepon A, Swaminathan A. Environmentally optimal, nutritionally sound, protein and energy conserving plant based alternatives to US meat. Sci Rep. 2019; 9: 10345. 
15. Shepon A, Eshel G, Noor E, Milo R. The opportunity cost of animal based diets exceeds all food losses. Proc Natl Acad Sci U S A. 2018; 115: 3804-3809.

16. Eshel G, Shepon A, Noor E, Milo R. Environmentally optimal, nutritionally aware beef replacement plant-based diets. Environ Sci Technol. 2016; 50: 8164-8168.

17. Willett W, Rockström J, Loken B, Springmann M, Lang T, Vermeulen S, et al. Food in the Anthropocene: The EAT-Lancet Commission on healthy diets from sustainable food systems. Lancet. 2019; 393: 447-492.

18. Melina V, Craig W, Levin S. Position of the academy of nutrition and dietetics: Vegetarian diets. J Acad Nutr Diet. 2016; 116: 1970-1980.

19. Sykes JT. The influence of climate on the regional distribution of nut crops in Turkey. Econ Bot. 1975; 29: 109-115.

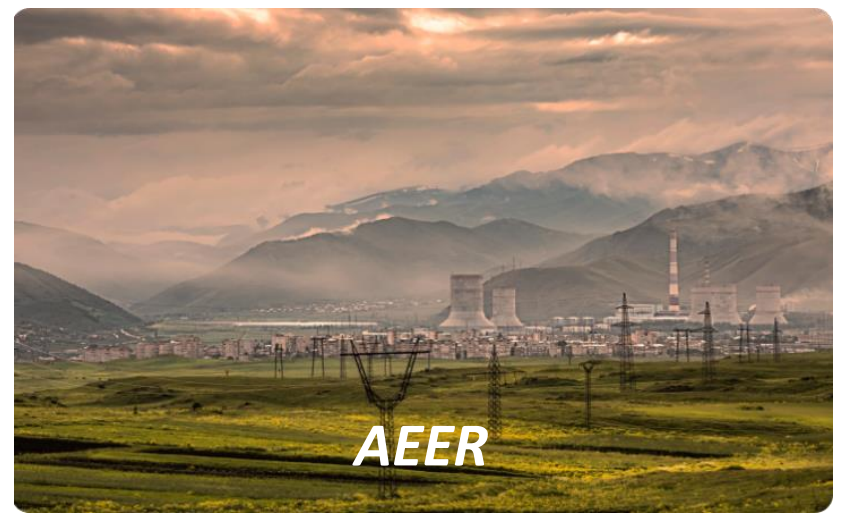

Enjoy $A E E R$ by:

1. Submitting a manuscript

2. Joining in volunteer reviewer bank

3. Joining Editorial Board

4. Guest editing a special issue

For more details, please visit:

http://www.lidsen.com/journals/aeer 